\title{
Significance of Lymph Node Dissection in Gynecological Oncology
}

\author{
Günter Emons
}

Klinik für Gynäkologie und Geburtshilfe, Georg-August-Universität Göttingen, Germany

\section{Keywords}

Lymph node dissection - Cervical cancer - Ovarian cancer . Endometrial cancer

\section{Summary}

Lymph node dissection has been an integral part of the surgical treatment of gynecological malignancies for over a century. The significance of lymph node dissection in gynecological oncology is reviewed in the light of our current knowledge of tumor biology. The original 'centrifugal theory' of metastasis formation leading to the concept of 'radical' surgery has its limitations. Lymph node dissection will still be necessary in gynecological oncology until molecular diagnostics have developed sufficiently and efficacious systemic therapies are available.

\section{Introduction}

During the last decades of the 19th century, the development of the classical surgical procedures in oncology were made possible by the advent of anesthesia and antisepsis. According to the anatomical and pathophysiological knowledge of the time, these procedures were based on the 'radical' strategy of removing the primary tumor, the surrounding tissue $($ radix $=$ root $)$ and the loco-regional lymph nodes [1]. The most prominent advocate of this cancer theory, termed 'centrifugal theory', was W. S. Halstedt [1], and his legacy remained part of the standard treatment for breast cancer surgery for 80 years. Surgical procedures based on the 'centrifugal theory' aiming at the radical removal of the primary tumor and the loco-regional lymph node basins were also developed in gy- necological oncology. In the famous text book 'Gynecological Operations', Döderlein perfectly depicted the lymphatic drainage system of the female genitalia (fig. 1) [2]. He described 3 main lymphatic basins: (1) from the vulva and the lower third of the vagina to the inguinofemoral nodes; (2) from the upper vagina and the cervix uteri via the parametria to the pelvic wall vessels; and (3) from the uterine corpus and the ovaries along the ovarian vessels to the paraaortic and paracaval basins between the renal vessels and the inferior mesenteric artery [2]. The removal of pelvic lymph nodes had become standard procedure in the surgical treatment of cervical cancer, and the extirpation of inguinofemoral nodes was recommended for the operation of vulvar cancer [2]. However, for endometrial cancer treatment, gynecological surgeons at that time could not remove the 'regional group of lymph nodes' in the paraaortic region for fear of causing potential injuries to the large blood vessels [3].

With the development of surgical procedures, including laparoscopic and robotic techniques, pelvic and paraaortic lymph node dissection (LND) became part of the surgical treatment of vaginal, cervical, endometrial, tubal, and ovarian cancer. In vulvar cancer, in most cases, LND is restricted to the inguinofemoral nodes. However, LNDs have been shown to result in intraoperative complications and long-term morbidity (e.g. [4]). The benefits of LND in gynecological cancers have either not been studied systematically or are not as obvious as the frequency of its use would suggest.

\section{Digression: The Example of Breast Cancer}

Due to the high incidence of breast cancer, much more evidence is available on the relevance of LND in this disease than in female pelvic cancers. As already mentioned, at the end of the 19th century, radical mastectomy, including axillary

\section{KARGER \\ Fax +497614520714 \\ Information@Karger.com}

www.karger.com

\section{(c) 2014 S. Karger GmbH, Freiburg}

2296-5270/14/0379-0500\$39.50/0

Accessible online at:

www.karger.com/ort
Prof. Dr. med. Günter Emons

Klinik für Gynäkologie und Geburtshilfe

Georg-August-Universität Göttingen

Robert-Koch-Str. 40, 37075 Göttingen, Germany

emons@med.uni-goettingen.de 


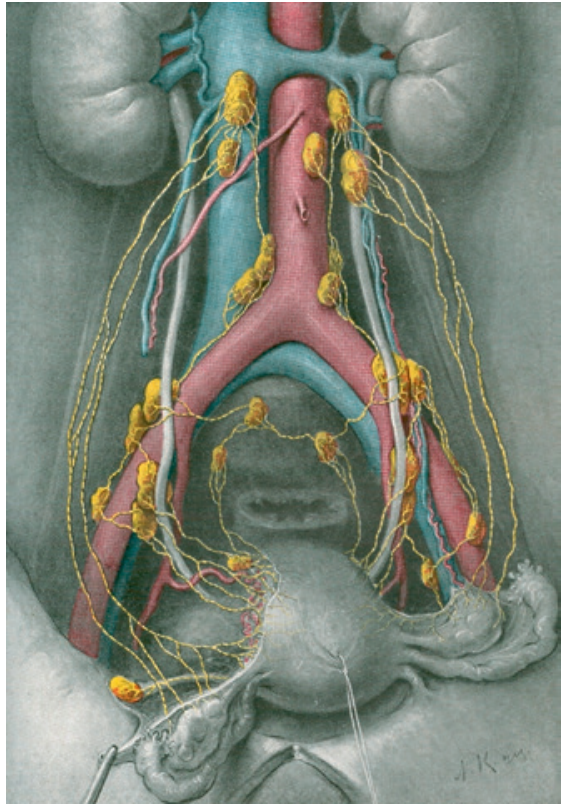

Lymph drainage of the female genitalia [2].

lymph nodes (levels 1-3), had become the standard of care [1]. Halstedt realized that 3-year survival was very low in patients with positive nodes [1], and the reaction of Halstedt and his school (including H. Cushing) was the extension of LND to the supraclavicular, the cervical and the mediastinal nodes. This strategy had its climax with the development of 'superradical mastectomies' with en bloc resection of ribs, part of the sternum and the internal mammary nodes [5]. The era of radical and super-radical mastectomies found its end with the NSABP-B04 trial, led by Bernhard Fisher (also a surgeon). Between 1971 and 1974, 1079 breast cancer patients (mean tumor diameter $3.3 \mathrm{~cm}$ ) with no palpable axillary metastasis were either treated with radical mastectomy (including LND) or simple mastectomy plus radiation of lymph nodes or simple mastectomy and axillary dissection only if palpable nodes developed [6]. As there were no differences in overall survival (even after 25 years), radical mastectomy seemed no longer to be a reasonable standard treatment. $40 \%$ of women having a radical mastectomy in this trial had positive axillary nodes. However, only $19 \%$ of patients having a simple mastectomy without any initial treatment of the axilla developed clinically apparent axillary metastases [6]. This meant that about 50\% of axillary non-palpable metastases regressed in the absence of any treatment. On the other hand, $19 \%$ of patients without any axillary treatment developed a relapse in the axillary nodes. Therefore, for many years primary axillary dissection became the standard of care, although a positive effect on overall survival was never shown $[6,7]$.

To reduce the morbidity associated with axillary LND, sentinel node biopsy was introduced, requiring a formal axillary dissection only when metastatic disease was found in the sentinel lymph node [7, 8]. In 2011, the American College of Surgeons published the Z0011 Trial in which 891 patients with T1-T2 breast cancers, no palpable axillary nodes, and 1-2 posi-
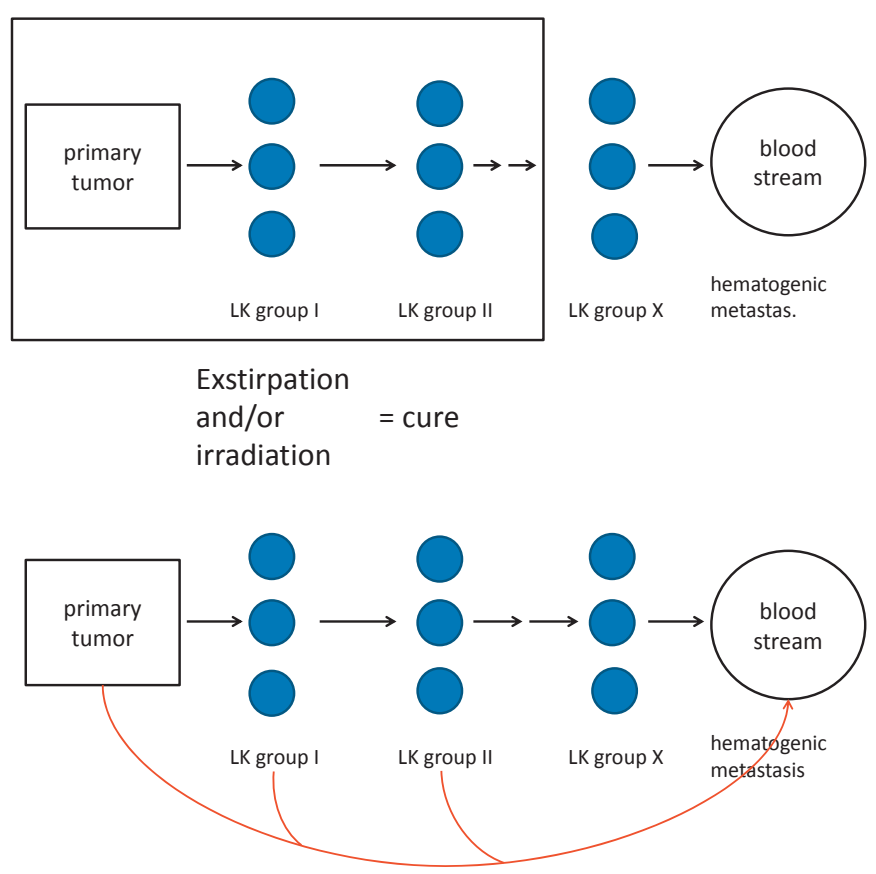

Exstirpation and/or irradiation $\neq$ cure but only local control $\Rightarrow$ an efficacious systemic therapy is warranted

Fig. 2. Hypothetical modes of metastasis. A 'Centrifugal theory'. B Simultaneous lymphatic and hematogenous metastases.

tive sentinel nodes were randomized to have a formal axillary LND or no further axillary treatment [7]. All patients had lumpectomy and tangential whole breast irradiation. $96 \%$ had adjuvant systemic therapy [7]. Axillary relapses were found in $0.5 \%$ (axillary dissections) and $0.9 \%$ (sentinel node dissection only). No differences in loco-regional recurrences, or diseasefree and overall survival were observed. Interestingly, $27.4 \%$ of the patients with an axillary dissection had additional metastatic nodes on histological examination. However, in the cohort in whom an exclusive sentinel node biopsy was performed only $0.9 \%$ of patients developed axillary relapse. This means that nearly all of the occult axillary metastases left after sentinel node biopsy had a complete remission on tangential whole breast irradiation and adjuvant systemic therapy [7]. The recent update of the Clinical Practice Guideline of the American Society of Clinical Oncology (ASCO) consequently recommends no axillary dissection for these patients [8].

Thus, we have gone a long way from the concept of Halstedt, via those of Cushing, Urban, and Fisher to our modern procedure: In most cases of breast cancer (typically the early stage of disease detected in the screening programs) only a lumpectomy plus tangential whole breast irradiation and a sentinel biopsy are performed. If the sentinel node is positive, a more intensive adjuvant systemic therapy is added, leading to an axillary relapse rate below $1 \%$. Thus, in breast cancer treatment - at least in early stage disease - LND has become only a diagnostic procedure, determining the extent of systemic adjuvant therapy. Positive axillary nodes indicate a 
high risk of hematogenous metastasis or disseminated disease. The surgical removal of affected lymph nodes is most probably not relevant, and radical or super-radical LND does not improve cure rates. The 'centrifugal theory', in which the radical extirpation of the primary tumor and the first lymph node groups provides cure (fig. 2A), does not apply to breast cancer. Rather, in parallel to lymphatic spread, there is hematogenous dissemination (fig. 2B). Extirpation and/or irradiation of lymph nodes only provide a local control. For cure, an efficacious systemic treatment is necessary.

\section{Gynecological Cancers}

What do we know about the significance of LND in gynecological cancers? In detail, we have to ask and answer the following questions:

(1) Has LND a prognostic significance?

(2) Is there an efficacious therapy for $\mathrm{pN} 1$ disease?

(3) Is the $\mathrm{pN}$ status essential for the indication of adjuvant therapy (predictive relevance)?

(4) Is LND per se therapeutic?

\section{Cervical Cancer}

LND has a clear prognostic significance in cervical cancer. In stage IB/IIA patients, 5-year disease-free survival was $>90 \%$ (pN0), 81\% (pN1, 1 pelvic lymph node metastasis), $41 \%$ (2-3 pelvic lymph node metastasis), $23 \% \geq 4$ pelvic lymph metastasis), or 30-35\% (paraaortic metastasis) [9]. The main reason for relapse was distant metastases. All patients had adjuvant radiation with or without chemotherapy. Thus, LND has a clear prognostic significance.

If a pN0 situation is confirmed, adjuvant radiochemotherapy can be avoided in most situations. When a pN1 status is found, most authors recommend no radical surgery but a primary radiochemotherapy. Positron emission tomographycomputed tomography (PET-CT), and if negative laparoscopic LND, have been used to evaluate the paraaortic lymph node status, and to determine the need for paraaortic irradiation [10]. The 'therapeutical' significance of surgically removing lymph node metastases has never been studied systematically in cervical cancer.

Thus, in cervical cancer, although LND has been traditionally performed for more than a century, its significance has not been formally investigated. In early stage disease, LND may help to select patients in whom radical hysterectomy should be performed. If $\mathrm{pN} 1$ disease is found, radiochemotherapy is the treatment of choice. In pN1 and higher stages of (pelvic) disease, paraaortic LND is recommended (if PET-CT is negative) to decide whether or not a paraaortic irradiation field is required.

Unfortunately, systemic therapy in cervical cancer is far less efficacious than in breast cancer, so that in cervical cancer, in spite of resection and/or irradiation of pelvic and paraaortic lymph nodes metastases, survival remains poor $[9,10]$.

\section{Vulvar Cancer}

LND has a clear prognostic significance in vulvar cancer. Lymph node metastases in the inguinofemoral region significantly worsen survival [11]. Adjuvant radiotherapy somewhat improves the prognosis of patients with pN1 disease [11]. Whether this can be further improved by additional chemotherapy remains to be shown. Surgical removal of affected inguinofemoral lymph nodes has a relevant therapeutic value, as survival is markedly decreased when these lymph nodes are not dissected, e.g. due to false-negative sentinel nodes [12].

\section{Ovarian Cancer}

LND has a clear prognostic significance in apparently early ovarian cancer. Here, positive lymph nodes lead to stage IIIC instead of I or II. In stages IIIC and higher, LND has no further impact on staging. A pN1 situation leads to the indication of adjuvant chemotherapy, which is rather efficacious in ovarian cancer. However, chemotherapy is not recommended for stages pT1a, b G1, pN0. Chemotherapy is recommended for stages pT1c and higher and in all G3 tumors, and should be considered in pT1a, b G2. Thus, only a small number of ovarian cancer patients really depend on LND for the indication of chemotherapy.

Is LND therapeutical in ovarian cancer? An analysis of the Surveillance, Epidemiology and End Results (SEER) database found a relevant improvement of cause-specific survival for stages I-IIIB. This was, however, largely due to stage migration: in pT1 cases up to $25 \%$ positive nodes, and in pT2 up to $30 \%$, were found, shifting them into stage IIIC [13]. Even in patients with stage IIIC or stage IV disease, LND had a positive impact on survival. The authors considered that thorough LND might reflect the quality of cytoreductive surgery [13]. In a randomized trial, no effects of systematic LND on overall survival of optimally debulked (residual tumor $\leq 1 \mathrm{~cm}$ ) ovarian cancer patients with stage IIIB, C and IV disease were found, although progression-free survival was improved from 22.4 to 27.2 months [14].

In a retrospective analysis of 1,942 ovarian cancer patients, $\mathrm{du}$ Bois et al. [15] found that in patients with no residual tumor after cytoreductive surgery, systematic LND significantly improved overall survival. They concluded that for patients with advanced ovarian cancer in whom complete intraperitoneal debulking can be achieved, systematic LND might offer an additional survival benefit. A prospective, randomized trial, checking this hypothesis has been performed and is now in follow-up [15].

\section{Endometrial Cancer}

With the improvement of surgical and anesthesiological techniques, systematic LND was recommended for all endometrial cancer patients [16]. It then became apparent that patients with stage I disease (endometrioid) had a disease-specific 5-year survival of $96 \%$ with or without LND [17]. Only stage I grade 3 cancers and those of stage $\geq$ II had a significant benefit from LND [17]. 
Two randomized trials evaluating pelvic LND in early stage endometrial cancer found it to have no impact on progression-free and overall survival [18, 19]. Although these trials had several weaknesses, it is now generally accepted that LND should not be performed in endometrioid endometrial cancer of stage pT1a, G1 and G2, as it only induces unnecessary morbidity and costs [20]. However, in a retrospective analysis [21], systematic pelvic and paraaortic LND up to the renal vessels was shown to provide a highly significant improvement in overall survival in cases with G3 and type 2 endometrial cancers (serous; clear cell) and in stages $\geq$ pT1b (myometrial invasion $\geq 50 \%$ ), indicating that LND did have a therapeutical impact in these patients. Patients with pN1 endometrial cancer (stage IIIC) have a poor prognosis. So far, no data are available showing that external beam radiotherapy has an effect on overall survival. Adjuvant systemic chemotherapy has some efficacy in improving progression-free and overall survival [22]. Large prospective trials are underway to assess the efficacy of adjuvant chemotherapy and adjuvant radiochemotherapy in high-risk endometrial cancer [23]. A large trial is in preparation to evaluate the predictive relevance of LND in high-risk endometrial cancer ( $\mathrm{pN} 0$ : no adjuvant therapy; $\mathrm{pN} 1$ : adjuvant therapy) [23]. Another trial is being prepared by the AGO-study group (Arbeitsgemeinschaft Gynäkologische Onkologie) to assess the therapeutical impact of systematic pelvic and paraaortic LND in high-risk endometrial cancer.

\section{Conclusions}

Halstedt's 'centrifugal theory' is not valid for cervical, vulvar, ovarian and endometrial cancers. As soon as lymph node metastases occur, the problem of distant metastases arises. Even the most thorough surgery and irradiation, although able to improve local control, cannot cure the disease in the absence of an efficacious systemic therapy. In breast cancer, systemic therapy is so powerful that even positive lymph nodes can be cured, and LND is increasingly losing importance. In ovarian cancer, chemotherapy is also efficacious, and can lead to a cure if all macroscopic tumor manifestations have been surgically removed. Ongoing trials must show whether LND of apparently non-enlarged nodes provides an additional benefit. In cervical cancer, a pre-therapeutical assessment of $\mathrm{pN}$ status might become a standard for aiding the decision of surgery versus radiochemotherapy, and the extent of the radiation field. In many endometrial cancers, LND is not indicated, as disease-specific survival is excellent with or without LND. In intermediate- and high-risk cases, randomized prospective trials are warranted. In vulvar cancer, LND is important as disease relapse in the groin is associated with high mortality. Trials are in preparation that will hopefully show whether adjuvant radiochemotherapy can improve survival.

In the future, it will be possible to define the molecular profile of a tumor from a biopsy, which will aid in designating a curative systemic therapy. LND in gynecological cancers will then be obsolete. For breast cancer, we are already approaching this stage of medicine. For cancers of the female genital organs, we still partly rely on LND, the concept developed more than a 100 years ago. We still need the prognostic value of the $\mathrm{pN}$ status, and in some cases also the improvement of local and perhaps systemic control obtained by LND. We should, however, refrain from performing LND just because it can be achieved so easily using laparoscopy or robotic techniques. We should only perform LND where it is medically indicated. We should also continue our efforts to asses these indications in high-quality clinical trials to avoid unnecessary morbidity.

\section{Disclosure Statement}

The author declares no conflict of interest.

\section{References}

1 Halstedt WS: The results of radical operations for the cure of breast. Ann Surg 1907;46:1-19.

2 Döderlein A: Carcinoma uteri; in Döderlein A, Krönig B (eds): Operative Gynäkologie, 4th ed. Leipzig, Thieme, 1921, pp. 574-670.

3 Stöckel W: Das Carcinoma uteri, in: Stoeckel W, Reifferscheid K (eds): Lehrbuch der Gynäkologie, 13th ed. Leipzig, Hirzel, 1924, pp. 337-380.

4 May K, Bryant A, Dickinson HO, et al.: Lymphadenectomy for the management of endometrial cancer. Cochrane Database Syst Rev 2010; (1):CD007585.

5 Urban JA: Management of operable breast cancer: The surgeon's view. Cancer 1978;42:2066-2077.

6 Fisher B, Jeong JH, Anderson S, et al.: Twenty-fiveyear follow-up of a randomized trial comparing radical mastectomy, total mastectomy, and total mastectomy followed by irradiation. N Engl J Med 2002;347:567-575.
7 Giuliano AE, Hunt KK, Ballman KV, et al.: Axillary dissection vs no axillary dissection in women with invasive breast cancer and sentinel node metastasis: A randomized clinical trial. JAMA 2011;305:569-575.

$>8$ Lyman GH, Temin S, Edge SB, et al.: Sentinel lymph node biopsy for patients with early-stage breast cancer: American Society of Clinical Oncology Clinical Practice Guideline Update. J Clin Oncol 2014; 32:1365-1383.

$\checkmark 9$ Sakuragi N: Up-to-date management of lymph node metastasis and the role of tailored lymphadenectomy in cervical cancer. Int J Clin Oncol 2007; 12:165-175.

10 Gouy S, Morice P, Narducci F, et al.: Prospective multicenter study evaluating the survival of patients with locally advanced cervical cancer undergoing laparoscopic para-aortic lymphadenectomy before chemoradiotherapy in the era of positron emission tomography imaging. J Clin Oncol 2013; 31:3026-3033.

11 Woelber L, Kock L, Gieseking F, et al.: Clinical management of primary vulvar cancer. Eur J Cancer 2011;47:2315-2321.

12 Oonk MH, van Hemel BM, Hollema H, et al.: Size of sentinel-node metastasis and chances of nonsentinel-node involvement and survival in early stage vulvar cancer: Results from GROINSS-V, a multicentre observational study. Lancet 2010;11: 646-652.

13 Rouzier R, Bergzoll C, Brun JL, et al.: The role of lymph node resection in ovarian cancer: Analysis of the Surveillance, Epidemiology, and End Results (SEER) database. BJOG 2010;117:1451-1458.

14 Panici PB, Maggioni A, Hacker N, et al.: Systematic aortic and pelvic lymphadenectomy versus resec- 
tion of bulky nodes only in optimally debulked advanced ovarian cancer: A randomized clinical trial. J Natl Cancer Inst 2005;97:560-566.

15 du Bois A, Reuss A, Harter P, et al.: Potential role of lymphadenectomy in advanced ovarian cancer: A combined exploratory analysis of three prospectively randomized phase III multicenter trials. J Clin Oncol 2010;28:1733-1739.

16 Neubauer NL, Havrilesky LJ, Calingaert B, et al.: The role of lymphadenectomy in the management of preoperative grade 1 endometrial carcinoma. Gynecol Oncol 2009;112:511-516.

17 Chan JK, Cheung MK, Huh WK, et al.: Therapeutic role of lymph node resection in endometrioid corpus cancer: A study of 12,333 patients. Cancer 2006;107:1823-1830.
Benedetti Panici P, Basile S, Maneschi F, et al.: Systematic pelvic lymphadenectomy vs. no lymphadenectomy in early-stage endometrial carcinoma: Randomized clinical trial. J Natl Cancer Inst 2008;100:1707-1716.

19 Kitchener H, Swart AM, Qian Q, et al.: Efficacy of systematic pelvic lymphadenectomy in endometrial cancer (MRC ASTEC trial): A randomised study. Lancet 2009;373:125-136.

20 Dowdy SC, Borah BJ, Bakkum-Gamez JN, et al.: Prospective assessment of survival, morbidity, and cost associated with lymphadenectomy in low-risk endometrial cancer. Gynecol Oncol 2012;127:5-10.
1 Todo Y, Kato H, Kaneuchi M, et al.: Survival effect of para-aortic lymphadenectomy in endometrial cancer (SEPAL study): A retrospective cohort analysis. Lancet 2010;375:1165-1172.

22 Johnson N, Bryant A, Miles T, et al.: Adjuvant chemotherapy for endometrial cancer after hysterectomy. Cochrane Database of Syst Rev 2011;(10): CD003175.

-23 Creutzberg CL, Kitchener HC, Birrer MJ, et al. Gynecologic Cancer InterGroup (GCIG) endometrial cancer clinical trials planning meeting: taking endometrial cancer trials into the translational era. Int J Gynecol Cancer 2013;23:1528-1534.

\section{Karger Journals in}
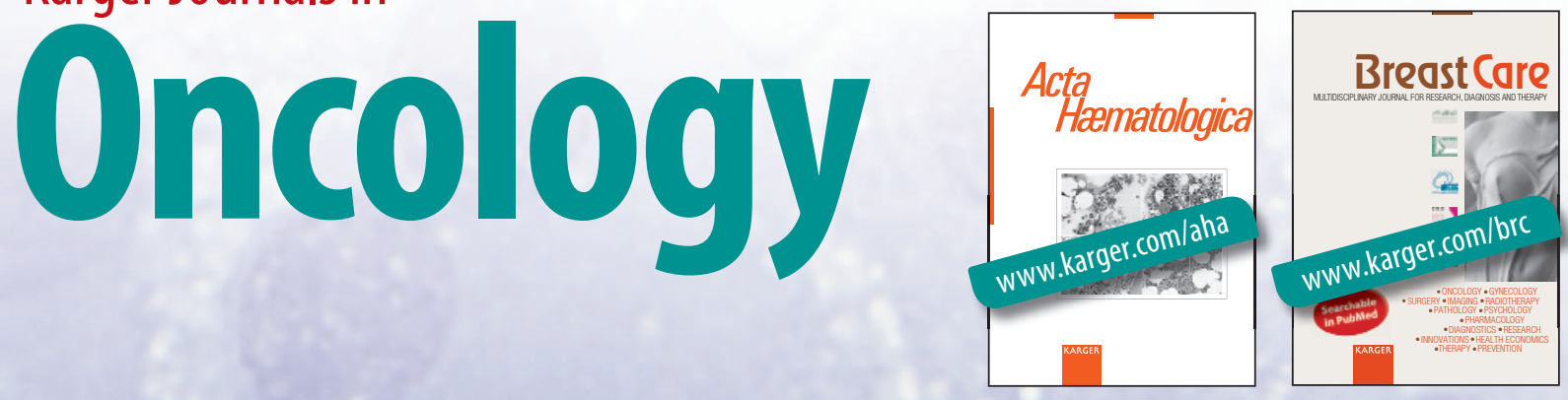

Find detailed information and read a free online sample on each journal's website!
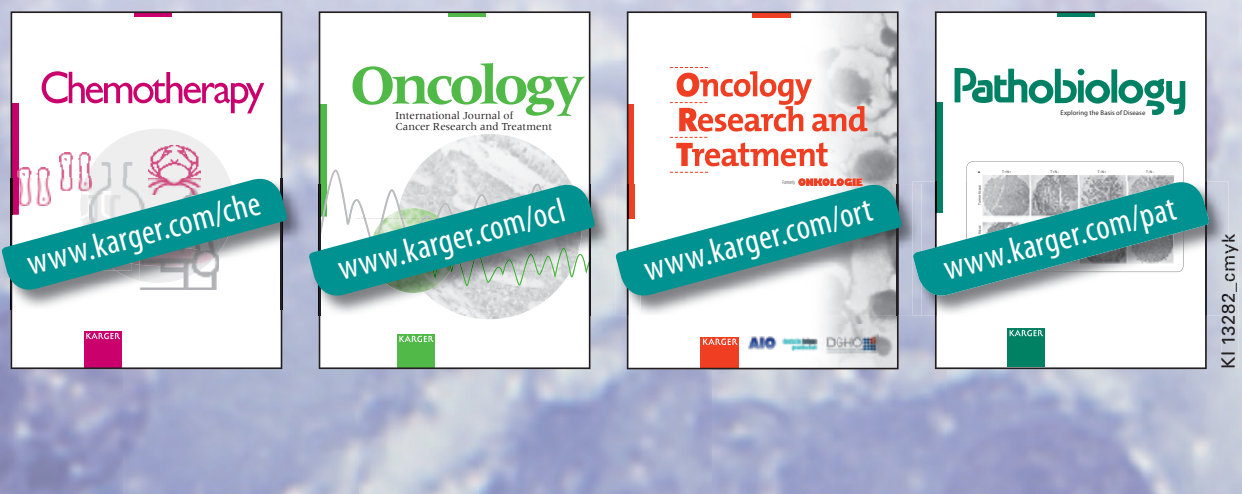